\title{
Higgs Pair Production via Vector Boson Fusion at the LHC
}

\author{
Andrea MASSIRONI* \\ Northeastern University \\ E-mail: andrea.massironiecern.ch
}

The measurement of Higgs pair production will be one of the cornerstones of the LHC physics program in the coming years, with the upcoming high-energy and high-luminosity phase. The Vector Boson Fusion (VBF) production channel probes directly the coupling of new physics and of Higgs to gauge bosons, in addition to reducing background contribution.

The production via VBF of an heavy object on-shell, subsequently decaying to a Higgs boson pair, may occur: the case of a KK-graviton from warped extra dimension models will be taken as a benchmark.

In addition, in the VBF channel, the production of Higgs pairs is sensitive to the strong interactions of a composite Higgs boson, and would allow a direct extraction of the hhVV quartic coupling. Other anomalous couplings of the Higgs to itself or to vector bosons would also lead to modified production rate and kinematics.

XXII. International Workshop on Deep-Inelastic Scattering and Related Subjects 28 April - 2 May 2014

Warsaw, Poland

* Speaker. 
The measurement of Higgs pair production will be one of the cornerstones of the LHC physics program in the coming years, with the upcoming high-energy and high-luminosity phase. Di-Higgs production is directly sensitive to the Higgs trilinear coupling, and then it provides information on the scalar potential responsible for electroweak symmetry breaking [1]. In addition, it is sensitive to the underlying strength of the Higgs interactions at high energies and it can test the composite nature of the Higgs boson [2, 3, 4].

In the Standard Model (SM), the dominant mechanism for the production of two Higgs bosons at the LHC is gluon fusion $[1,5,6,7,8]$, with a cross section of approximately $40 \mathrm{fb}[9,10,11,12$, $13,14]$ computed at next-to-next-to leading order at a center-of-mass energy of $\sqrt{s}=14 \mathrm{TeV}$. The second production mechanism is the Vector Boson Fusion (VBF), with a cross section of about $1 \mathrm{fb}$ at $\sqrt{s}=14 \mathrm{TeV}$, computed at next-to-next-to leading order $[5,8,15,16,17,18,19,20]$. The coupling of new physics and of Higgs to gauge bosons can be probed by looking at VBF production, and, in addition, VBF topology allows a reduction of the background contamination maintaining high signal efficiency. A schematic view of a VBF di-Higgs event is shown in Figure 1.

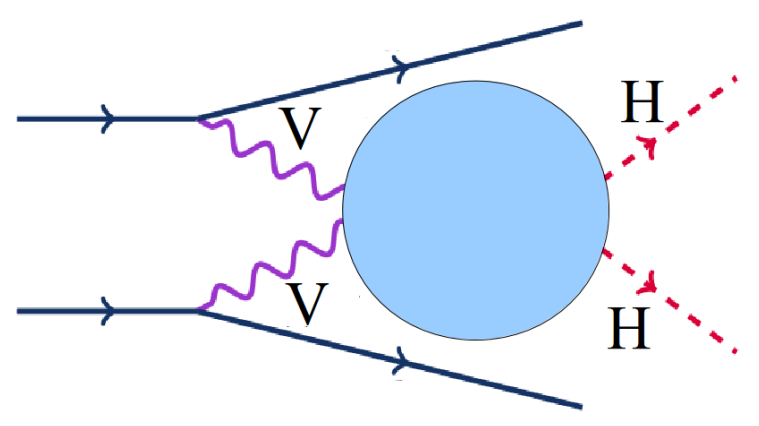

Figure 1: Diagram for VBF production of two Higgs bosons.

Higgs pair production can be substantially enhanced in various BSM scenarios, mainly through two mechanisms:

- production of an on-shell resonance decaying into two Higgs bosons

- new physics at higher scales, that is observable indirectly through modification of the Higgs couplings

In the following, two separate studies at parton level concerning the two approaches for new physics searches are described. Final states with the largest branching fractions are considered: $h h \rightarrow 4 b$ and $h h \rightarrow 2 b 2 W$.

The results are based on [21].

\section{Resonant VBF di-Higgs search}

(Work in collaboration with Alexander Belyaev, Olivier Bondu, Alexandra Oliveira, Rogerio Rosenfeld and Veronica Sanz)

Resonant Higgs pair production at the LHC in VBF tests the couplings of the gauge bosons to new physics and therefore it is important as a test to any new model. A model of warped 
extra dimensions where a KK-graviton $(G)$ (see [22] and references within) can be produced onshell through vector boson fusion and subsequently decays into a pair of Higgs bosons is used as benchmark. Although the KK-graviton production cross-section in VBF is about 1/10 of gluon fusion production, given the high branching ratio of the KK-graviton into two Higgs bosons ( 25\% for masses greater than $500 \mathrm{GeV}$ ) and given the efficient background rejection thanks to VBF topology selections, a high signal yield is expected with a reasonable background contamination. The analysis is performed at parton level. Only $\mathrm{h} \rightarrow \mathrm{bb}$ decay processes are considered, exploiting the high $\mathrm{h} \rightarrow \mathrm{bb}$ branching ratio. Acceptance cuts on the jets are defined requiring jet $\mathrm{p}_{T}>30$ $\mathrm{GeV}$ and $|\eta|<4$.7. In addition, the VBF topology is enhanced by means of a selection on the invariant mass of the di-jet system $\left(\mathrm{m}_{j j}>400 \mathrm{GeV}\right)$. No cut on the pseudorapidity separation between the VBF jets $\Delta \eta_{j j}$ is applied, due to $\mathrm{p}_{T}$-dependence of the KK-graviton vertices with vector bosons [23].

In order to exploit the boosted topology from $\mathrm{h} \rightarrow$ bb decays the transverse momentum of the diHiggs system is required to have $\mathrm{p}_{T}^{h h}>60 \mathrm{GeV}$ and the pseudorapidity separation between the two Higgs bosons to be $\Delta \eta_{h h}<2$. The higher the $G$ mass the higher is the probability for the two Higgs bosons to be boosted and the two b-jets to be merged: a uniform analysis efficiency combining different categories is recovered [24], as shown in Figure 2.

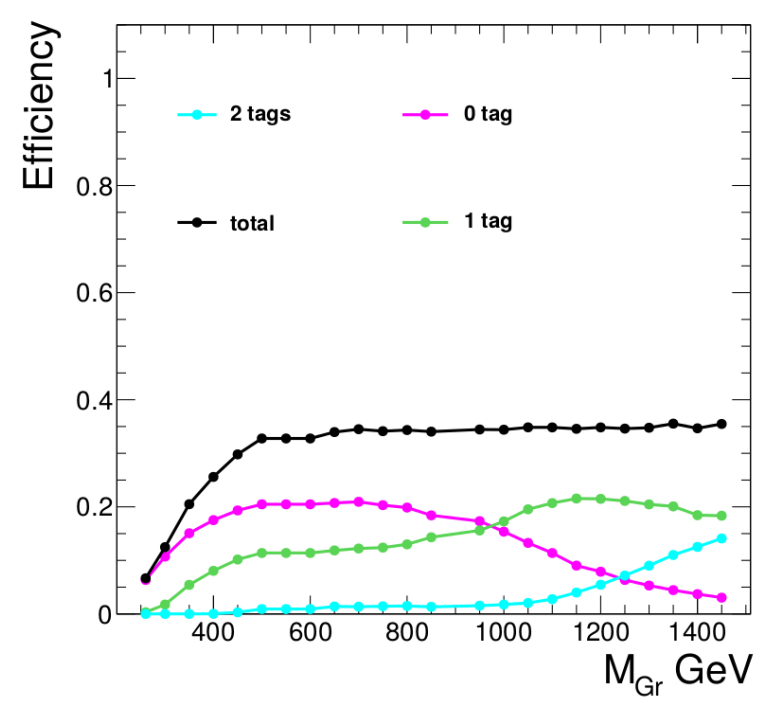

Figure 2: Signal efficiency separation based on the number of boosted hard systems after the analysis flow. A uniform signal efficiency across the $G$ mass range is recovered by means of the combination of different categories on the number of boosted reconstructed objects.

Perfect b-tag efficiency is considered within experimental acceptance volume. No selections concerning the $h \rightarrow b b$ and $G \rightarrow h h \rightarrow b b b b$ invariant masses are applied, and are left to be addressed in a dedicated study with showering and reconstruction effects, that is currently ongoing, also considering different additional final states.

A simple cut based analysis is performed and with the high-luminosity LHC data, $3 \mathrm{ab}^{-1}$ at $13 \mathrm{TeV}$, high signal yields are expected (from tens to hundreds of events depending on the $G$ mass range). 
Although the current selection efficiencies for the background are less than 5\%, a still important background contamination is present, but it can be further reduced by means of selections based on the invariant mass of the di-b system $m_{b b}$ and the 4 b-jets final state $m_{4 b}$.

\section{Non-resonant VBF di-Higgs search at parton level (Work in collaboration with Olivier Bondu, Roberto Contino, Maxime Gouzevitch, Juan Rojo)}

An indirect search for new physiscs has been performed according to an effective lagrangian approach up to dimension 6 operators [4].

Three coupling parameters have been modified:

- $\mathrm{h}$ to VV vertex, $c_{V} g_{h V V}^{S M}$, that will be partially constrained through single Higgs searches;

- hh to VV vertex, $c_{2 V} g_{h h V V}^{S M}$, that is only measurable in a VBF hh process;

- $\mathrm{h}$ to hh vertex, $c_{3} g_{h h h}^{S M}$, that is accessible also via gluon fusion production of two Higgs bosons.

Two different di-Higgs decay final states are considered: $\mathrm{hh} \rightarrow 4 \mathrm{~b}$ and $\mathrm{hh} \rightarrow 2 \mathrm{~b}+\mathrm{WW}(2 \ell 2 v)$. Jets and leptons are considered only within detector acceptances: light and $\mathrm{b}$ jets are required to have $\mathrm{p}_{T}>25 \mathrm{GeV}$ and light (b) jet $|\eta|<4.5$ (2.5), while leptons $|\eta|<2.5$. In order to select the VBF topology, the invariant mass of the di-jet system is required to have $\mathrm{m}_{j j}>500 \mathrm{GeV}$ and a high separation between the two jets $\Delta \mathrm{R}_{j j}>4.0$ is demanded. This first feasibility study is performed at parton level.

For the $4 b+2 j$ final state the main QCD backgrounds are QCD $4 b+2 j$ and $Z(b b)+2 b+2 j$ production. Tighter VBF cuts are applied in order to suppress QCD background, namely $\mathrm{m}_{j j}>$ $800 \mathrm{GeV}$.

For the $2 b+W W(2 \ell 2 v)$ final state the main background is $W W+2 b+2 j$ SM production, that is mainly dominated by $t \bar{t}+2 \mathrm{j}$ events. In order to reject this background, the $\mathrm{h} \rightarrow \mathrm{WW}$ kinematics has been exploited: the invariant mass of the di-lepton system is required to have $\mathrm{m}_{l l}<70 \mathrm{GeV}$, and the transverse mass of the di-W system, defined in the following equation 2.1, to have $\mathrm{m}_{T}<$ $125 \mathrm{GeV}$.

$$
m_{T}(W W) \equiv\left(\left(\sqrt{m_{l l}^{2}+\left|\vec{p}_{T l l}\right|^{2}}+\sqrt{m_{l l}^{2}+\left|\vec{p}_{T m i s s}\right|^{2}}\right)^{2}-\left|\vec{p}_{T l l}+\vec{p}_{T m i s s}\right|^{2}\right)^{1 / 2},
$$

In addition, in both analyses, the invariant mass of the di-b system is required to be around the Higgs boson one: $\mathrm{m}_{b b}=125 \mathrm{GeV} \pm 15 \%$ for both bb pairs.

The expected event numbers for SM di-Higgs VBF production are very small, but for $\left(c_{V}-c_{2 V}\right) \neq 0$ the $\mathrm{VV} \rightarrow$ hh cross section $(\mathrm{V}=\mathrm{W} / \mathrm{Z}$ ) grows with the partonic energy, thus leading to an enhancement up to a factor one hundred with respect to SM expectations in the high-energy regime, as shown in Figure 3. In the $4 b+2 j$ final state, in order to access the high-energy regime, the invariant mass $4 \mathrm{~b}$ jets is required to be $\mathrm{m}_{4 b}>1000 \mathrm{GeV}$, while for the $2 \mathrm{~b}+\mathrm{WW}(2 \ell 2 v)$ final state the invariant mass of the $2 \mathrm{~b}$ jets +2 leptons is demanded to be $\mathrm{m}_{l l b b}>500 \mathrm{GeV}$ and the transverse momentum of the di-b system to have $\mathrm{p}_{T}^{b b}>200 \mathrm{GeV}$. 


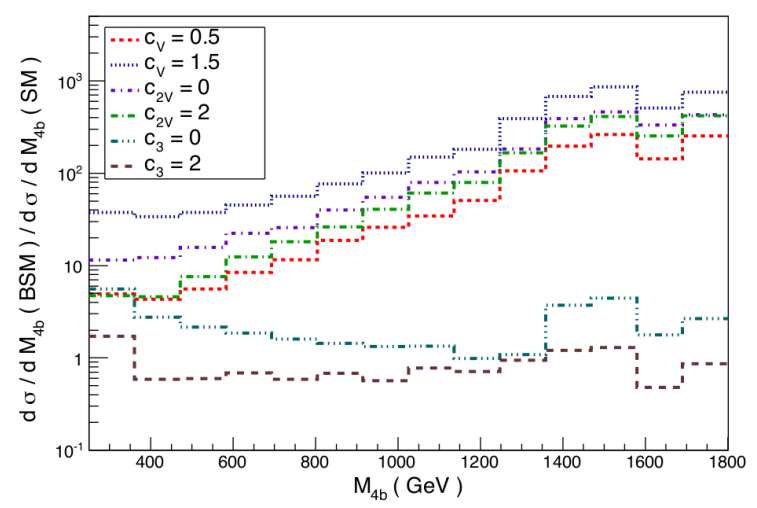

Figure 3: Ratio of the differential cross sections with respect to the $4 \mathrm{~b}$ invariant mass between the case with modified couplings and the one with SM couplings, as a function of the $4 \mathrm{~b}$ invariant mass. An enhancement by up to a factor one hundred with respect to SM expectations is present in the high-energy regime for modifications of the $c_{V}$ and $c_{2 V}$ couplings, while no effect in the high-energy regime is present for $c_{3}$, as expected, since its contribution is more visible in the threshold regime $\left(2 \mathrm{~m}_{h}\right)$.

After all the selections have been applied, the signal efficiency is quite high ( $25 \%)$ for the non-SM VBF hh signal, leading to a signal over background ratio bigger than one for BSM scenarios. Nonetheless, the high-luminosity LHC (HL-LHC) is needed, $\mathrm{L}=3 \mathrm{ab}^{-1}$, given the low cross section of this process.

\section{Conclusions}

Vector Boson Fusion di-Higgs production will be a probe for new physics, whose access can be either direct, looking for resonances decaying into two Higgs bosons, or indirect, looking at deviations from SM predictions of couplings involving the Higgs boson.

The results reported for the HL-LHC show good signal efficiency and yield for heavy resonances in the mass range between $250 \mathrm{GeV}$ and $1 \mathrm{TeV}$.

At the HL-LHC the sensitivity prospects to the hhVV $\left(c_{2 V}\right)$ vertex seem promising, given the high signal over background ratio and the high signal efficiency for the non-SM VBF hh process.

\section{References}

[1] A. Djouadi, W. Kilian, M. Muhlleitner, and P.M. Zerwas. Production of neutral Higgs boson pairs at LHC. Eur.Phys.J., C10:45-49, 1999.

[2] R. Grober and M. Muhlleitner. Composite Higgs Boson Pair Production at the LHC. JHEP, 1106:020, 2011.

[3] G.F. Giudice, C. Grojean, A. Pomarol, and R. Rattazzi. The Strongly-Interacting Light Higgs. JHEP, 0706:045, 2007.

[4] Roberto Contino, Christophe Grojean, Mauro Moretti, Fulvio Piccinini, and Riccardo Rattazzi. Strong Double Higgs Production at the LHC. JHEP, 1005:089, 2010. 
[5] Oscar J.P. Eboli, G.C. Marques, S.F. Novaes, and A.A. Natale. TWIN HIGGS BOSON PRODUCTION. Phys.Lett., B197:269, 1987.

[6] EW Nigel Glover and Jochum J Van der Bij. Higgs boson pair production via gluon fusion. Nuclear Physics B, 309(2):282âĂŞ294, 1988.

[7] T. Plehn, M. Spira, and P.M. Zerwas. Pair production of neutral Higgs particles in gluon-gluon collisions. Nucl.Phys., B479:46-64, 1996.

[8] J. Baglio, A. Djouadi, R. Grober, M.M. Muhlleitner, J. Quevillon, et al. The measurement of the Higgs self-coupling at the LHC: theoretical status. JHEP, 1304:151, 2013.

[9] S. Dawson, S. Dittmaier, and M. Spira. Neutral Higgs boson pair production at hadron colliders: QCD corrections. Phys.Rev., D58:115012, 1998.

[10] Daniel de Florian and Javier Mazzitelli. Higgs Boson Pair Production at Next-to-Next-to-Leading Order in QCD. Phys. Rev. Lett. 111,, 201801:201801, 2013.

[11] Jonathan Grigo, Jens Hoff, Kirill Melnikov, and Matthias Steinhauser. Higgs boson pair production at the LHC: top-quark mass effects at next-to-leading order. PoS, RADCOR2013:006, 2013.

[12] Jonathan Grigo, Jens Hoff, Kirill Melnikov, and Matthias Steinhauser. On the Higgs boson pair production at the LHC. Nucl.Phys., B875:1-17, 2013.

[13] Daniel de Florian and Javier Mazzitelli. Two-loop virtual corrections to Higgs pair production. Phys.Lett., B724:306-309, 2013.

[14] R. Frederix, S. Frixione, V. Hirschi, F. Maltoni, O. Mattelaer, et al. Higgs pair production at the LHC with NLO and parton-shower effects. Phys.Lett., B732:142-149, 2014.

[15] Wai-Yee Keung. Double Higgs From $W-W$ Fusion. Mod.Phys.Lett., A2:765, 1987.

[16] Duane A. Dicus, Kalpana J. Kallianpur, and Scott S.D. Willenbrock. Higgs Boson Pair Production in the Effective W Approximation. Phys.Lett., B200:187, 1988.

[17] Kalpana J. Kallianpur. Pair Production of Higgs Bosons via Heavy Quark Annihilation. Phys.Lett., B215:392, 1988.

[18] A. Abbasabadi, W.W. Repko, Duane A. Dicus, and Roberto Vega. Comparison of Exact and Effective Gauge Boson Calculations for Gauge Boson Fusion Processes. Phys.Rev., D38:2770, 1988.

[19] A. Dobrovolskaya and V. Novikov. On heavy Higgs boson production. Z.Phys., C52:427-436, 1991.

[20] Ling Liu-Sheng, Zhang Ren-You, Ma Wen-Gan, Guo Lei, Li Wei-Hua, et al. NNLO QCD corrections to Higgs pair production via vector boson fusion at hadron colliders. 2014.

[21] G. Brooijmans, R. Contino, B. Fuks, F. Moortgat, P. Richardson, et al. Les Houches 2013: Physics at TeV Colliders: New Physics Working Group Report, ARXIV:1405.1617;. 2014.

[22] Hyun Min Lee, Myeonghun Park, and Veronica Sanz. Gravity-mediated Dark Matter. Eur.Phys.J., C74:2715, 2014.

[23] A. Djouadi, R.M. Godbole, B. Mellado, and K. Mohan. Probing the spin-parity of the Higgs boson via jet kinematics in vector boson fusion. Phys.Lett., B723:307-313, 2013.

[24] Maxime Gouzevitch, Alexandra Oliveira, Juan Rojo, Rogerio Rosenfeld, Gavin P. Salam, et al. Scale-invariant resonance tagging in multijet events and new physics in Higgs pair production. JHEP, 1307:148, 2013. 